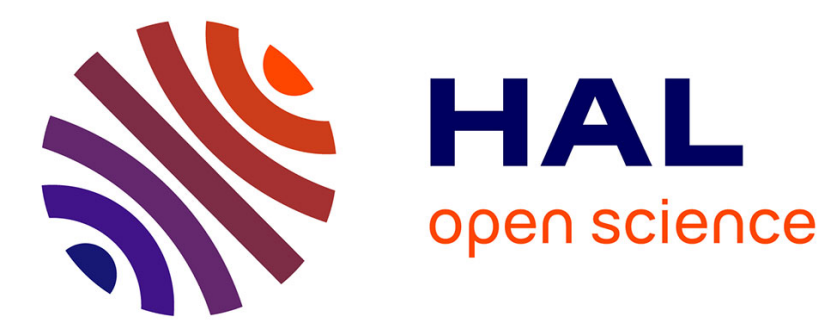

\title{
Experimental investigation of bubble and drop formation at submerged orifices
}

\author{
Nicolas Dietrich, Nadia Mayoufi, Souhila Poncin, Huai-Zhi Li
}

\section{To cite this version:}

Nicolas Dietrich, Nadia Mayoufi, Souhila Poncin, Huai-Zhi Li. Experimental investigation of bubble and drop formation at submerged orifices. Chemical Papers, 2013, 67 (3), pp.313 - 325. 10.2478/s11696-012-0277-5 . hal-01268183

\section{HAL Id: hal-01268183 \\ https://hal.science/hal-01268183}

Submitted on 18 Jul 2021

HAL is a multi-disciplinary open access archive for the deposit and dissemination of scientific research documents, whether they are published or not. The documents may come from teaching and research institutions in France or abroad, or from public or private research centers.
L'archive ouverte pluridisciplinaire HAL, est destinée au dépôt et à la diffusion de documents scientifiques de niveau recherche, publiés ou non, émanant des établissements d'enseignement et de recherche français ou étrangers, des laboratoires publics ou privés. 


\title{
EXPERIMENTAL INVESTIGATION OF BUBBLE AND DROP FORMATION AT SUBMERGED ORIFICES
}

\author{
N. DIETRICH ${ }^{*}, 1,2$, N. MAYOUFI ${ }^{1}$, S. PONCIN ${ }^{1}$ and Huai Z. LI ${ }^{1}$
}

\section{Laboratory of Reactions and Process Engineering, Nancy-Université, CNRS, 1 rue} Grandville, 54000 Nancy, France.

2. Université de Toulouse, INSA, Laboratoire d'Ingénierie des Systèmes Biologiques et des Procédés, INRA-CNRS, 135 avenue de Rangueil, 31077 Toulouse, France.

* To whom correspondence should be addressed.

Tel.: +33(0)5 615597 81, Fax: +33 (0)5 615597 60, E-mail: dietrich@,insa-toulouse.fr

Keywords: bubble, drop, formation, PIV measurements

\section{Abstract}

The aim of this study is to investigate bubble/drop formation at a single submerged orifice in stagnant Newtonian fluids and to gain a qualitative understanding of the formation mechanism. The effects of various governing parameters were studied. The formation behaviours of bubble and drop in Newtonian aqueous solutions were investigated experimentally under different operating conditions with various orifices. The results show that the volume of detached dispersed phase increases with viscosity, surface tension, orifice diameter and dispersed phase flow rate. A PIV system was employed to measure quantitatively the velocity flow field during the bubble/drop formation, giving interesting information useful for the elucidation of the fundamental formation process at the orifice. It was revealed that the orifice shape has a strong influence on formed bubble size. Furthermore, based on a simple mass balance, a global correlation was proposed to predict both bubble and drop size with good agreement. 


\section{Introduction}

37 The formation of gas bubbles or liquid drops is an important fundamental phenomenon that conditions significantly the hydrodynamics in gas-liquid and liquid-liquid reactors commonly used in chemical and petrochemical processes, cosmetics, mineral processing, etc. The contact between phases is generally achieved either by bubbling gas into the liquid or by making droplets in another liquid in various applications such as absorption, distillation, emulsification, froth flotation, etc.

In most of the multiphase equipment, the knowledge of the transport and transfer processes across a gas-liquid or liquid-liquid interface is useful for the estimation of interfacial area, mass transfer coefficient and dispersed holdup. Furthermore, the physicochemical properties of the liquid phase (viscosity, surface tension, density, etc.) as well as the characteristics of the dispersed phase (bubble/drop size, bubble/drop rise velocity, etc.) govern the hydrodynamics and flow pattern in the system. The formation mechanism determines the primitive bubble or drop size in the system and has logically important consequences on the hydrodynamics.

The earliest reports on the formation of single bubbles and drops were published by Tate (1864) and Bashforth \& Adams (1883). Bashforth and Adams computed the shape of liquid menisci for equilibrium conditions and published their results in the form of tables. From their data, the evolution of the bubble or drop volume can be calculated in the quasi-steady regime.

57 Different models of varying complexity have been proposed including viscous and inertial forces. Davidson and Schüler (1960a and 1960b) presented models for dynamical bubble formation at submerged orifices for the case of viscous and inviscid liquids. A significant 60 amount of works on the bubble formation at submerged orifices over a wide range of 
operating parameters have been published in the literature in the last few decades. Ouz and Prosperetti (1993) investigated the dynamic bubble growth at a needle. The importance of the chamber volume under a submerged orifice was pointed out by Marmur and Rubin (1976). Kumar and Kuloor (1970) published an exhaustive report on bubble formation under a wide range of operating conditions. Tsuge (1986) reviewed the hydrodynamics of bubble formation from submerged orifices and discussed various models for the bubble formation mechanism. Rabiger and Vogelpohl (1986) briefly discussed the various parameters that affect the bubble formation. Since then, bubble formation receives continuous attention in the literature, for example, Jamialahmadi et al., 2001; Li et al., 2002; Kulkarni and Joshi, 2005; Buwa et al., 2007. Most of these studies can be classified on the basis of the operating conditions for the gas phase, such as constant flow rate or constant pressure. However, the observations of many investigators are not concordant because of variation induced by the physical properties of the gas-liquid systems, the type of nozzles and the operating parameters (gas velocity, system pressure, etc.). Hence, more observations are required to clarify the bubble formation process.

Bubble and drop formation from a nozzle or an orifice have been extensively studied from theoretical and experimental perspectives as mentioned before. Many studies were devoted to drop formation in a continuous fluid (Null and Johnson, 1958; Narasinga et al. ;1966; Schele and Bernard, 1968; Chazal and Ryan, 1971; Hamad et al. 2001) and correlations were proposed based on the properties of the fluids studied (density, viscosity, surface tension) and on the operating conditions (diameter of the opening, flow of the dispersed phase) in order to predict the drop volume. The theoretical analysis has been primarily based on macroscopic force balances assuming that drop formation occurs in two stages. The first stage is the static growth of the drop, which ends when the forces are no longer in equilibrium. The second stage corresponds to the necking and breaking of the drop from the nozzle (Scheele and 
Meister, 1968; Heertjes et al., 1971). From these studies, it can be concluded that the volume of the drops depends not only on the nozzle size and liquid properties, but also on the liquid flow rate through the nozzle (unlike the pendant drop case (Michael, 1981).

These studies mainly differ in how the authors approach the second formation stage. The simplified model gives a good approximation for the drop size when the flow rate is small but deviations from the experimental measurements occur when the flow rate becomes greater (Clift et al. 1978; Kumar and Kuloor, 1970). Although the previously cited studies of drop formation provide some interesting features of the phenomenon, there is still few information on the fundamental formation process.

To improve our understanding of the bubble and drop formation mechanism, significant information could be obtained from Particle Image Velocimetry (PIV) measurements. While a great deal of research has focused on rising bubbles in Newtonian and non-Newtonian fluids using PIV measurements (Funfschilling and Li, 2001), few experimental studies concern such measurements for the bubble formation. Scarano (1997) studied bubble formation in a Newtonian fluid with a large bubble wake masking the flow circulation, Li et al. (2001) studied bubble formation in non-Newtonian fluids during the expansion stage at an orifice. To our best knowledge, complete velocity flow field measurements for the bubble or drop formation have never been reported in the literature yet. A thorough knowledge of bubble and drop size evolution and break-up is necessary for complete prediction and design of practical processes that involve interfacial phenomena and flows between two phases. In this study, a PIV system was used to obtain this original information and gain new light into this subject. 
111 The experimental setup used to investigate bubble and drop formation under constant flow

112 conditions is shown in Fig. 1. The experiments were conducted in a square PMMA column

113 (inner dimensions: $0.1 \mathrm{~m}$ wide, $0.5 \mathrm{~m}$ high) filled with aqueous phases. The gas bubbles and

114 the organic liquid drops were generated through a submerged orifice located at the bottom of

115 the central section of the column. Two types of orifices were used (a plate orifice of $0.01 \mathrm{~m}$

116 and a thin needle). Various orifice diameters $\left(0.5\right.$ to $\left.4 \times 10^{-3} \mathrm{~m}\right)$ were investigated in this study.

118 A capillary edge of $0.2 \mathrm{~mm}$ was used to avoid the wetting problem at the orifice. All

119 experiments were carried out at a constant ambient temperature of 293K. A Rheometric Fluid

120 Spectrometer RFS II (Rheometric Scientific, USA) was employed to measure the rheological

121 properties. The surface and interfacial tensions of the solutions were measured using a

122 Tracker tensiometer (I.T. Concept, France) for gas-liquid and liquid-liquid systems. The

123 physical properties of these systems used are reported in Tables 1 and 2.

124

125

Table 1. Fluid properties

126

\begin{tabular}{c|c|c|c} 
Fluid & density kg.m & viscosity Pa.s & Tension force $\times 10^{-3}{\mathrm{~N} . \mathrm{m}^{-1}}^{-3}$ \\
\hline Air & 1.15 & $1.8 \times 10^{-5}$ & - \\
\hline Water & 998 & $1 \times 10^{-3}$ & 72.5 \\
\hline HV45 50\% wt & 1030 & 0.205 & 37.5 \\
HV45 65\% wt & 1050 & 0.515 & 38.5 \\
\hline Silicon oil 5 & 950 & $6 \times 10^{-3}$ & 18.5 \\
Silicon oil 10 & 953 & $11 \times 10^{-3}$ & 20.2 \\
Silicon oil 50 & 955 & $55 \times 10^{-3}$ & 20.3 \\
Silicon oil 100 & 960 & 0.11 & 20.4
\end{tabular}

127

128

129

130 
Table 2. Interface properties (Surface or interfacial tension $\times 10^{-3} \mathrm{~N} \cdot \mathrm{m}^{-1}$ )

\begin{tabular}{c|c|c|c|c} 
Fluid & Silicon oil 5 & Silicon oil 10 & Silicon oil 50 & Silicon oil 100 \\
\hline Water & 24.2 & 22.1 & 20.2 & 18.5 \\
\hline HV45 50\% wt & 18.8 & 15.2 & 13.4 & 11.8 \\
\hline HV45 65\% wt & 18.2 & 14.9 & 13.1 & 11.2
\end{tabular}

133 Dimensionless numbers, such as Bond (buoyancy and tension force), Reynolds (inertia and

134 viscous forces), and Weber (inertia and tension forces) numbers were used to characterize the 135 gas and liquid flow:

$$
B o=\frac{d_{\mathrm{int}}^{2}\left(\rho_{l}-\rho_{g}\right) g}{\sigma}
$$

$$
\operatorname{Re}=\frac{\rho_{d}\left(\frac{4 Q}{\pi d_{\text {int }}^{2}}\right) d_{\text {ext }}}{\mu d}
$$

$$
W e=\frac{\rho d\left(\frac{4 Q}{\pi d_{\text {int }}^{2}}\right)^{2} d_{\text {ext }}}{\sigma}
$$

The phenomenon was recorded with a high-speed digital video camera (CAMRECORD 600, Germany) at a rate of 1000 images per second. Images contained $1280 \times 1024$ pixels

144 displaying grey levels. The lighting was provided by a $800 \mathrm{~W}$ halogen lamp which 145 illuminated the column in an indirect way on a white screen placed behind the column. The 146 image sequences obtained were then analysed in order to calculate the position, velocity and 147 shape of the dispersed phase. Instantaneous velocity fields around a forming bubble or a drop 148 were measured by means of a Particle Image Velocimetry technique (PIV, Dantec Dynamics,

149 Denmark) (Fig. 1). Illumination sheets were generated with two pulsed Nd-YAG LASERS 150 (SOLO-I-15 PIV New Wave Research, USA) arranged side-by-side and crossed the vertical 151 symmetry axis of the dispersed phase. The energy produced by this source was $2 \times 15 \mathrm{~mJ}$. 152 These lasers emitted green light with a wavelength of $532 \mathrm{~nm}$ for a duration of 8 to $10 \mathrm{~ns}$. The 
153 time between the flashes varied from less than one microsecond to a few milliseconds. The

$154 \mathrm{Nd}$-Yag lasers were also designed to ensure a sequence of illuminations at a frequency of 15

155 Hz. The laser beams first crossed a cylindrical lens which gave a laser sheet of strong light

156 intensity and small thickness (2.5 mm maximum). They were focused and superimposed on

157 one zone of measurement. The size of the seeding particles was chosen so that the particles

158 followed the flow correctly and produced sufficient light to make the recording possible and

159 minimize the errors on measurements. In general, seeding particles with diameters of 10 to 50

160 micrometers were used for liquid phases. In this study, they were composed of silvered glass

161 microspheres of sizes ranging from 10 to 30 micrometres (average diameter of 15

162 micrometres) and of a density of $1400 \mathrm{~kg} \cdot \mathrm{m}^{-3}$. The size was determined to minimize both

163 Brownian movement and seeding particle sedimentation. The camera, placed perpendicular to

164 the laser sheets, took two successive images at the maximum intensity of the laser pulse.

165 These images were divided into a few thousand small interrogation areas of $16 \times 16$ pixels. A

166 cross-correlation was then performed between the corresponding interrogation areas. When

167 the flow was correctly inseminated, the measurement errors on the velocities were less than

$1685 \%$. From these experiments, the liquid velocity flow field around a forming bubble or drop

169 were obtained at an orifice.

170 


\section{Experimental results}

172 The experiments were chosen in the aim of obtaining quantitative information on the 173 dynamics of bubble and drop formation.

\subsection{Bubble formation}

176 The shape evolution of bubbles with time during the process of bubble growth and 177 detachment in aqueous solutions is shown in Fig. 2. It can be clearly seen that, in all the cases studied of bubble formation in water or HV45 solutions, bubbles expanded spherically at the beginning, then evolved from a spherical shape to an inverse teardrop shape symmetrically, 180 with the vertical axis passing through the orifice centre. The first shape was due to the

181 importance of surface tension at the beginning of bubble formation. Subsequently, the influence of bubble buoyancy gradually became important as the bubble grew: the interface moved upwards and the bubble was elongated to an obvious teardrop shape. Changing viscosities or physical properties modified the shape but not the global formation mechanism.

Further information about the formation mechanism could be obtained from the measurement of flow fields. The major difficulty in applying the PIV technique arose due to the light reflection at the interface. The light reflected at the bubble interface is usually much more intense than the light scattered by the tracer particles, which prevents the registration of their

190 images on the recording media near the interface. For a big orifice, light reflection and 191 obscurity near the bubble, due to its shadow, lead to a lack of information on the right side of

192 the flow field. Thus only orifices of diameter smaller than $4 \times 10^{-3} \mathrm{~m}$ were used so as to avoid 193 this problem. The experimental flow fields obtained during the formation of a $180 \times 10^{-9} \mathrm{~m}^{3}$

194 bubble in water are shown in Fig. 3. The time between two successive frames was $5 \times 10^{-3} \mathrm{~s}$. 195 The choice of this time depends on the rapidity of the physical phenomena. The time between 
196 two successive recordings was about $66 \times 10^{-3} \mathrm{~s}$ and the final formation time was about $0.3 \mathrm{~s}$.

197 At $t / t_{\mathrm{f}}=0.03$ (Fig. 3a) and $\mathrm{t} / \mathrm{t}_{\mathrm{f}}=0.3$ (Fig. 3b), the expansion phase of the bubble formation can

198 be observed. At the start of bubble expansion, an upward flow was firstly observed at the

199 front of the bubble, which pushes the liquid (Fig 3a). As the bubble grew, the upward flow

200 increased until the amount of liquid moved reached a critical value and the liquid fell on the

201 sides of the bubble front (Figs. 3b and 3c). The downward flow thus created resulted in conservation of mass and caused the circulation region at the side of the bubble. In water or viscous solutions, bubble and velocity profiles were perfectly axis-symmetric, and displayed neither shape nor trajectory oscillations within the range of bubble sizes studied before detachment. Fig. $3 \mathrm{~d}$ shows the velocity profile a few milliseconds before the gas film rupture and bubble detachment $\left(\mathrm{t} / \mathrm{t}_{\mathrm{f}}=0.99\right)$. The combination of the upward and downward flow led to the formation of axis-symmetric circulation loops. The space corresponding to the recirculation region expanded while the bubble volume increased. The recirculation was due to the elongation phase of the bubble and created a shear exerted at the neck of the elongated bubble, leading to its final rupture. At $\mathrm{t} / \mathrm{t}_{\mathrm{f}}=1.15$ (Fig. 4e), the formation cycle ended with the

211 break-up of the neck of the bubble and its release into the column. Velocity field magnitude increased with the bubble velocity, and an upward flow appeared in the wake of the bubble due to mass conservation and recirculation. The final flow field was in agreement with that

214 reported by previous studies and PIV measurement of bubble motion in fluids (Li et al. 2001;

215 Frank et al. 2006; Dietrich et al. ;2008; and Dietrich et al. 2010). 
The detached bubble volumes were correlated as a function of flow rate for capillaries of different diameters and different systems. The effect of the gas flow rate on the bubble growth is shown in Fig.4. Obviously, the bubble volume increases gradually with the gas flow rate. The effect of the orifice diameter on the bubble volume at detachment from the orifice is plotted in Fig. 4a. It can be observed that the bubble volume changes sharply with the diameter of the orifice due to the increase of both drag and surface tension with the orifice diameter, as both forces effectively prevent quick bubble detachment from the orifice. As a result, the bubble growth time becomes longer and the bubble volume increases accordingly.

Figure $4 \mathrm{~b}$ shows the relationship between the volume of the bubble at detachment from the orifice and the gas flow rate in solutions with different viscosities and surface tension. It is well known that the surface tension acting on the bubble tends to restrain the growth of the bubble. The increase of HV45concentration in water led to an increment of viscosity which resulted in the increase of the viscous drag. Hence the bubble experimented more difficulty in detaching from the orifice. Consequently, bubble formation was prolonged and the volume of 236 the bubble increased. The bubble broke finally away from the orifice because of the continuous gas input in the bubble.

Concerning the orifice shape, two configurations were tested as shown on Fig 1. Contrary to 240 what is reported in the literature, orifice shape has a non-negligible influence. The effect of 241 the shape of the orifice on the bubble volume at detachment is plotted in Fig. 5.

243 For all the diameters studied, the bubble volume was greater for bubbles formed on a thin 244 orifice rather than a plate orifice. It can be observed that this effect was particularly stronger 245 for small orifice diameter. Firstly, the wetting on the orifice was thought to be responsible. 
246 But if this were true, the bubble should would be bigger in the case of the plate orifice.

247 Because this was not so, wettability was discarded and local hydrodynamic effects should be 248 envisaged.

250 The use of the PIV system to measure the velocity field around the two different orifice 251 shapes could give further information to elucidate this phenomenon. The liquid velocity field around a growing bubble in stagnant water can be observed in Fig. 6 for the two injection systems. Two flow rates used were exactly the same. Clearly, the bubble was bigger in the case of the thin orifice (Fig. 6a) rather than the plate orifice (Fig 6b), confirming then the volume evolution shown in Fig 5.

The two velocity flow fields showed similar global tendency but, for the plate orifice, recirculation regions caused a supplementary breakup mechanism at the neck of the bubble. The circulation loops seemed to be stopped on the orifice. In contrast, , the recirculation

261 caused mainly the bubble elongation with the thin orifice. This effect could be less marked for 262 bigger orifices.

263 Many parameters such as flow conditions, orifice size and shape, and physical properties were

264 tested. The results show that the bubble volume increased with the liquid viscosity, surface 265 tension, orifice diameter and gas flow rate.

\subsection{Drop formation}

268 The setup used to form drops was the same as for bubble formation. But it consisted 269 essentially of a fine capillary tube through which the oil phase was delivered at a constant 270 volumetric flow rate. The plate orifice was not used because of serious wetting problems

271 occurring during drop formation. A liquid drop was formed directly from the tip of the tube. 
The capillary tubes were made of stainless steel and had tips that were polished and machined flat to allow the drop to wet the front surface completely.

In order to avoid any wetting problems with the oil phase, the drop formation was studied only at several sizes of thin orifice: $0.5,1,2,3$ and $4 \times 10^{-3} \mathrm{~m}$ respectively. When a viscous oil was injected continuously through an orifice into quiescent, immiscible aqueous phase under low flow rates, oil phase broke up as discrete drops directly from the tip of the nozzle under buoyancy as shown in Fig. 7. It shows a temporary picture sequence obtained by the high-

280 speed camera during the experiments for a drop forming in quiescent water from a tube of $4 \times 10^{-3} \mathrm{~m}$, at a constant liquid flow rate of $10^{-9} \mathrm{~m}^{3} \mathrm{~s}^{-1}$. As the drop volume increased with the continuous oil alimentation, the drop rose on the edge of the orifice. The drop shape gradually changed from quasi-spherical to teardrop during the growth as shown by Zhang (1999). When

284 the size of the drop became sufficiently large, the buoyancy force exceeded the surface tension force (at $\mathrm{t} / \mathrm{t}_{\mathrm{f}}=0.75$ ), the drop necked quickly and an important part started to detach from the orifice. During this sequence (necking), the drop elongated quickly and contracted in the middle to create a liquid thread that connected the drop and the liquid on the orifice (Zhang 1999). This thread was stretched with the fast rise of the drop and broke at its downer end, resulting in a free primary drop. After the thread breakup, the cone-shaped liquid remaining on the orifice tended to retrieve its apex and became spherical because of the capillary force. Typically this rupture at both ends of the thead lead to the creation of a satellite small droplet, having a volume less than $1 \%$ of that of the main drop.

294 The velocity field measured by PIV is shown in Fig. 8. Due to the lower speed of the 295 expansion phase of the growing drop, it was very difficult to measure a velocity profile with 296 precision at these velocities. However, it can be supposed that velocity profiles in the 
expansion phase were the same as for bubble formation. The drop expansion should firstly create an upward flow at its front as a result of the drop pushing the liquid. As in the case of bubbles, the upward flow increased until the quantity of liquid moved reached a critical value and the liquid descended along the sides of the drop. The combination of the upward and

301 downward flows led to the formation of axis-symmetric circulation loops as shown in Fig. 8a.

302 As seen in Fig. 8b, the recirculation region expanded as the drop volume increased. This 303 recirculation region was a consequence of the drop elongation leading to its rupture (Fig 8c). At the moment of rupture, a thin thread of liquid linked the drop and the orifice. The formation cycle ended with the breakup of the drop neck and its release into the contineous phase. The magnitude of the liquid velocity field increased with the drop velocity, and an upward flow appeared in the wake of the drop due to mass conservation and recirculation. This final flow field is quite comparable to the bubble formation.

Fig. 9 focuses on the velocity profile when the thread broke, leading to the formation of a 311 secondary drop. The velocity flow fields before and after the detachment of a drop are 312 represented for a $250 \times 10^{-9} \mathrm{~m}^{3} \cdot \mathrm{s}^{-1}$ drop of HS100 silicone oil in water (orifice size $3 \times 10^{-3} \mathrm{~m}$ ).

314 Before the rupture, the flow fields around the thread displayed complex features: partly 315 upward flow behind the drop and partly directed downward. Fig. 9 illustrates the stretching 316 effects before and after the rupture of the liquid thread behind the neck of the drop. The downward and upward flows stretched the thread in opposed directions, leading to subsequent 318 rupture, which explains why the rupture occurs in the middle of the fluid thread and creates 319 satellite droplets due to interfacial instability. The velocity flow fields before and after the 320 rupture shown in Fig. 9 corroborate the shape evolution of the drop. 
322 Fig. 10 represents the volume evolution of formed drops for several systems used. Firstly, the 323 influence of the orifice size was investigated for a silicone oil drop in water. The results for 324 different orifice sizes tested are reported in Fig. 10a. As for bubble formation, the bigger the 325 orifice, the greater was the drop volume. However, the volume evolution with the flow rate of 326 dispersed phase differed from that for the bubble. For drop formation with a small orifice, the 327 volume increased with the flow rate until a maximum value. Thereafter, the volume decreased. This maximum was observed in other systems using different silicone oil (HS50 or HS10) as shown in Fig. 10b. The tendency was the same for these three systems. This maximum volume was observed by Narasinga (1964). The analysis by means of the high-

331 speed camera could explain this phenomenon. In Fig. 10b, three formation sequences were 332 plotted with the increase of the flow rate. The higher the flow rate, the longer was the neck behind the drop. The formation time was thus shorter at high flow rate than at low flow rate.

334 PIV measurements provided quantitative information about this phenomenon. The velocity 335 fields around a forming drop were quite different according to a low or a high flow rate (Fig. $33611)$.

338 The wake created by the preceding drop exerts a strong suction effect on the early stages of 339 the forming drop at orifice (Fig. 11b), and cannot be neglected as in many theoretical models 340 developed previosuly. This effect becomes smaller for the lower flow rate (Fig. 11a). The 341 wake effect leads to a shorter drop formation time and, indeed, a smaller volume of the drop 342 at detachment.

\subsection{Global correlation}

345 Even if the shape evolution is not exactly the same for drop and bubble formation, the 346 formation mechanism is quite similar for the systems studied. This study was carried out 
under large ranges of experimental conditions wit a total of 250 data points: bubble formation

348 in water and viscous fluids with several orifice sizes $\left(0.5\right.$ to $\left.4 \times 10^{-3} \mathrm{~m}\right)$ and several flow rates;

349 drop formation for several silicon oils (HS100, HS50 and HS10) in water and viscous fluids,

350 with several orifice sizes $\left(0.5\right.$ to $\left.4 \times 10^{-3} \mathrm{~m}\right)$ and several flow rates. To establish a comparison,

351 it is interesting to propose a correlation for the dispersed phase volume at detachment. If

352 inertia and viscous force are neglected (static model hypothesis) in the formation process, the

353 only two forces acting on forming bubbles or drops at orifice are the buoyancy

$354 P_{A}=\left(\rho_{l}-\rho_{g}\right) \cdot g . V$ and the capillary force $F_{c}=\sigma \cdot \pi \cdot d_{\text {int.cos}} \theta$. The global force balance leads to

355 Equation (4):

$$
\frac{d}{d_{\mathrm{int}}}=\left(\frac{6 \sigma}{d_{\mathrm{int}}{ }^{2}\left(\rho_{l}-\rho_{g}\right) g}\right)^{1 / 3}
$$

The number of bubbles or drops $\mathrm{N}_{\mathrm{b}, \mathrm{d}}$ is defined on the basis of the Bond number, equivalent diameter of dispersed phase and orifice internal diameter as follows:

$$
N_{b, d}=\frac{d}{d_{\text {int }}} B o^{\frac{1}{3}}
$$

362 As shown in Eq. 4, this number is equal to $\mathrm{N}_{0}=6^{1 / 3} \approx 1.81$ under static conditions. But this value is obtained for a system where inertia and viscous drag are neglected, which is not really the case for some of our experimental systems, e.g. formation in HV45 50\% or viscous silicone oil. In order to take into account these forces, a correction factor could be added, using Reynolds and Weber numbers and viscosity ratio between two phases, and interpreting the deviation between the static model and experimental data. The obtained correlation in Equ. 6 gives a good prediction of the bubble and drop volume.

$$
\frac{N_{b, d}}{N_{0}}=1+\frac{W e^{0.4}}{\operatorname{Re}^{0.43}}\left(\frac{\mu_{c}}{\mu_{d}}\right)^{0.38}
$$

Fig. 12 represents the experimental $\mathrm{N}_{\mathrm{b}, \mathrm{d}}$ versus the correlated one. The average error between experiment data and the correlation is less than $11 \%$. Thus, the correlation can be used for the 
prediction of the formed volume of a dispersed phase under various conditions: Reynolds

373 number ranging from $10^{-3}$ to $10^{3}$, orifice sizes between 0.5 to $4 \times 10^{-3} \mathrm{~m}$, stagnant phase

374 viscosity from 1 to $500 \mathrm{mPa} . \mathrm{s}$, and dispersed phase viscosity from $1.8 \times 10^{-5}$ to $10^{-1}$ Pa.s.

375 Nevertheless, this correlation is only valid for the thin orifice when no wetting phenomenon is

376 observed on the edge of the orifice. When wetting and spreading are observed, especially for

377 drop formation, the formation process and measured volume are not reproducible. The

378 influence of the orifice material and shape could lead to large errors in volume estimation and

379 require still more investigation.

\section{Conclusion}

382 The present work allows a better understanding of the formation mechanism of bubble or

383 drop. Flow field measurements obtained by PIV contribute to the flow quantification of the

384 different steps in continuous phase encountered during the formation and provide new light

385 into the interactions between successive drops or the influence of the orifice shape on the flow

386 pattern around a forming bubble or drop. The visualisation of the bubble/drop shape and

387 volume by the high-speed camera along with the flow fields by PIV would be useful for 388 modelling the formation mechanism and validating numerical approaches of the formation 389 process, in particular the thread rupture in the presence of interfacial instability. Finally, a 390 global correlation for bubble and drop formation was proposed to estimate the volume at 391 detachment from an orifice. However, this correlation is only valid in the absence of wetting 392 problems at orifice.

\section{Acknowledgements}

395 The financial assistance provided by the French Ministère de l'Education Nationale, de la 396 Recherche et de la Technologie is gratefully acknowledged. 


\section{Notation}

399

$400 \quad \mathrm{~N}_{\mathrm{b}, \mathrm{d}} \quad$ bubble/drop number

401 Re Reynolds number

402 Bo Bond number

403 We Weber number

$404 \mathrm{~V}$ bubble/drop volume, $\mathrm{m}^{3}$

$405 \mathrm{~d}$ bubble/drop equivalent diameter, $\mathrm{m}$

$406 \mu \quad$ fluid viscosity, Pa.s

$407 \mathrm{~g}$ gravity, $\mathrm{m} . \mathrm{s}^{-2}$

$408 \sigma \quad$ surface or interfacial tension, N. ${ }^{-1}$

409

410 Subscripts

411 c continuous phase

412 d dispersed phase

413 int inlet diameter

414 o equilibrium

415

416 


\section{Literature Cited}

418 Badam V. K., Buwa, V. and Durst F., 2007, "Experimental investigations on regimes of 419 bubble formation on submerged orifices under constant flow conditions", Canadian Journal of 420 Chemical Engineering, 85, 257-267.

421 Chao-Tai Chen, Jer-Ru Maa, Yu-Min Yang and Chien-Hsiang Chang (2001), "Drop 422 formation from flat tip nozzles in liquid-liquid system", Int. Comm. in Heat and Mass 423 Transfer, 28, 5, 681-692

424 De Chazal, L.E.M. and J.T.Ryan, (1971). "Formation of organic drops in water". AIChE J., 425 17,1226-1229.

426 Dietrich N., Poncin S. and LI H.Z, (2010). Dynamical deformation of a liquid-liquid interface, 427 Experiments in fluids, 2010, accepted

428 Dietrich N., Poncin S., Pheulpin S. and LI H.Z, (2008). Passage of a bubble through a liquid429 liquid interface. AICHE J. 2008, 54, 3, 594-600.

430 Frank X., Funfschilling D., Midoux N. \& Li H.Z. (2006). Bubbles in a viscous liquid: Lattice 431 Boltzmann simulation and experimental validation. J. Fluid Mech., 546, 113-122

432 Funfschilling D. and Li H.Z., Flow of non-Newtonian fluids around bubbles: PIV 433 measurements and birefringence visualisation, (2001). Chemical Engineering Science, 56, 434 1137-1141

435 Gaddis E. et Vogelpohl A. (1986). Bubble formation in quiescent liquids under constant flow 436 conditions. Chem. Eng. Sci., 41(1), 97-104.

437 Gerlach D., G. Biswas, F. Durst and V. Kolobaric (2005), "Quasi-static bubble formation on 438 submerged orifices", Int. J. Heat Mass Transfer, 48, 425-438.

439 Harkins W.D. and F.E. Brown (1919), "Determination of surface tension and the weight of 440 falling drops: The surface tension of water and benzene by the capillary height method", J. 441 Am. Chem. Soc., 41, 499.

442 Hayworth, C.B. and R.E. Treybal (1950). "Drop formation in two liquid phase systems".

443 Hibiki T. and M. Ishii (2007), " Lift force in bubbly flow systems", Chem. Eng. Sci., 62, 444 6457-6474

445 Horvath M., L. Steiner and S. Hartland (1978), "Prediction of drop diameter, hold-up and 446 backmixing coefficients in liquid-liquid spray columns." Can. J. Chem. Eng., 56, 9-15.

447 Jamialahmadi, M., M.R. Zehtaban, H.M. Steinhagen, A. Sarrafi and J.M. Smith (2001), 448 "Study of Bubble Formation under Constant Flow Conditions", Trans IChemE, 79 A, 523$449 \quad 532$ 
450 Kagan S.Z., Y.N. Kovalev and A.P. Zakharychev (1973), Theor. Osn. Chem. Eng. (Moscow), $4517,514$.

452 Klee A.J. and R.E. Treybal (1956), "Rate of rise or fall of liquid drops". A.I.Ch.E. J1.2/4 , $453 \quad 444-447$.

454 Krevelen van D.W. and P.J. Hoftijzer (1950), "Studies of gas bubbles formation", Chem Eng. 455 Prog., 46, 1, 1950.

456 Kulkarni A.A. and J. B. Joshi (2005), "Bubble Formation and Bubble Rise Velocity in Gas457 Liquid Systems: A Review", Ind. Eng. Chem. Res., 44, 5873-5931

458 Kumar R. and N.R. Kuloor (1970), "The formation of bubbles and drops", Adv. Chem. Eng., 459 Academic Press, N.Y.

460 Li H. Z., Frank, X. et Funfschilling, D. and Mouline Y. (2001). Towards the understanding of 461 bubble interactions and coalescence in non-Newtonian fluids: a cognitive approach. Chemical 462 Engineering Science, 56, 6419-6425.

463 Li H.Z., Mouline Y. and Midoux N., (2002). Modelling the bubble formation dynamics in 464 non-Newtonian fluids, Chem. Eng. Sci. 57, 339-346

465 Miyahara, T., and T. Takahashi, "Bubble Volume in Single Bubbling Regime with Weeping 466 at a Submerged Orifice,’’ J. Chem. Eng. Jpn., 17, 5971984.

467 Nahra H.K. and Y. Kamotani (2003), "Prediction of bubble diameter at detachment from a 468 wall orifice in liquid cross-flow under reduced and normal gravity conditions", Chem. Eng. 469 Sci., 58, 55-69.

470 Narasinga Rao E.V., R. Kumar and N.R. Kuloor (1966), "Drop Formation Studies in Liquid471 Liquid Systems", Chem. Eng. Sci., 21, 867-880.

472 Null H.R. and H.F. Johnson (1958), "Drop Formation in Liquid-Liquid Systems from Single 473 Nozzles", AIChE J., 4, 273-281.

474 Oguz H.N. and A. Prosperetti (1993), "Dynamics of bubble growth and detachment from a 475 needle", J. Fluid Mech., 257, 111-145.

476 Richards J.R., A.N. Beris and A.M. Lenhoff (1995), " Drop formation in liquid-liquid systems 477 before and after jetting", Phys. Fluids 7, 2617-2630.

478 Scarano, F.: Improvements in PIV image processing application to a backward facing step; 479 VKI PR 1997-01

480 Scheele G. and B. Meister (1968a), "Drop formation at low velocities in liquid-liquid 481 systems", Part I: "Prediction of drop volume", AIChE J., 14, 9-15.

482 Scheele G. and B. Meister (1968b), Part II: "Prediction of jetting velocity", AIChE J., 14, 16- 
19.

484 Steiner L. and S. Hartland (1983), "Hydrodynamics of Liquid-liquid Spray Columns" in 485 "Handbook of Fluids in Motion", ch. 40, N. Cheremisinoff and R. Gupta Ed., Ann Arbor 486 Science.

487 Tomotika S. (1935), "On the instability of a cylindrical thread of a viscous liquid surrounded 488 by another viscous fluid.” Proc. R. Sot. London Ser. A 150, 322.

489 Tsuge, H. (1986). Hydrodynamics of Bubble Formation from Submerged Orifices. 490 Encyclopedia of Fluid Mechanics, Vol. 3, Gulf Publishing, Houston, p. 191.

491 Tyler E. (1933), Instability of liquid jets, Philos. Mag. 16, 504-516.

492 Wong H., D. Rumschitzki and C. Maldarelli (1998), "Theory and experiment on the low493 Reynolds-number expansion and contraction of a bubble pinned at a submerged tube tip", J. 494 Fluid Mech., 356, 93-124.

495 Wraith A.E. (1971), "Two-stage bubble growth at a submerged plate orifice", Chem. Eng. 496 Sci., 26, 1659-1709.

497 Zhang, X.. "Dynamics of drop formation in viscous flows", Chemical Engineering Science, 498199906

499

500

501 